\title{
Driver Assistance System with a Dual Control Scheme: Effectiveness of Identifying Driver Drowsiness and Preventing Lane Departure Accidents
}

\author{
Yuichi Saito, Member, IEEE, Makoto Itoh, Member, IEEE, and Toshiyuki Inagaki, Senior Member, IEEE
}

\begin{abstract}
Driver drowsiness is a common cause of fatal traffic accidents. In this study, a driver assistance system with a dual control scheme is developed; it attempts to perform simultaneously the safety control of the vehicle and identification of the driver's state. The assistance system implements partial control in the event of lane departure and gives the driver the chance to voluntarily take the action needed. If the driver fails to implement the steering action needed within a limited time, the assistance system judges that "the driver's understanding of the given situation is incorrect", and executes the remaining control. We used a driving simulator equipped with the assistance system to investigate the effectiveness of identifying driver drowsiness and preventing lane departure accidents. Twenty students participated in three trials on a straight expressway, and they were required to implement only lateral control. We hypothesized that a participant cannot implement the action needed to maintain safety when he/she falls asleep, and that in such a case, the assistance system will implement the safety control repeatedly. The assistance system assisted the participants only when almost really needed, such as when their eyelids were closed. The results validated the hypothesis, showing that the assistance system implemented the safety control repeatedly when a participant fell asleep. In addition, the algorithms used by the assistance system to determine whether the driver can continue driving were evaluated through a leave-one-out cross-validation, and they were proven to be effective for identifying driver drowsiness.
\end{abstract}

Index Terms-Safety, advanced driver assistance systems, dual control, adaptive automation, driver behavior monitoring.

\section{INTRODUCTION}

$\mathrm{D}$ river drowsiness is a common cause of fatal traffic accidents. The National Highway Traffic Safety Administration (NHTSA) [1] reported that drowsy driving was involved in $2.2-2.6 \%$ of the total fatal crashes annually during the period 2005 through 2009. Several studies have reported that many sleep-related vehicle accidents occur during the periods of around 2:00-6:00 am and 14:00-16:00 pm [2-9], and it is often pointed out that night shifts make drivers particularly vulnerable [10].

Manuscript received June 26, 2015; revised October 30, 2015, January 17, 2016, and March 2, 2016; accepted March 21, 2016.

Yuichi Saito was with Graduate School of Systems and Information Engineering, University of Tsukuba, 305-8573 Tsukuba, JAPAN. He is now with the Department of Mechanical Systems Engineering, Tokyo University of Agriculture and Technology, 184-0012 Tokyo, Japan (e-mail: y-saito@cc.tuat.ac.jp).

Makoto Itoh is with Graduate School of Systems and Information Engineering, University of Tsukuba, 305-8573 Tsukuba, Japan (e-mail: itoh.makoto.ge@u.tsukuba.ac.jp).

Toshiyuki Inagaki is Vice President \& Executive Director, University of Tsukuba, 305-8573 Tsukuba, Japan (e-mail: inagaki.toshiyuki.gb@un.tsukuba.ac.jp)
Based on a model of automation [11], advanced driver assistance systems (ADAS) can be categorized into four classes: (a) enhancing perception, (b) arousing attention to potential risks, (c) setting off a warning, and (d) performing safety control [12]. Thus, ADAS are designed to complement driver capabilities for perception, cognition, action selection, and action implementation in a dynamic environment [13]. One way to reduce the number of sleep-related accidents could be to develop proactive safety technologies that detect driver drowsiness and provide the drivers with appropriate assistance.

Driver monitoring systems can be classified into two groups [14], namely, systems that monitor drivers by means of direct driver-related measurements, and those that monitor drivers by means of indirect driving-related measurements. Direct driver-related measurements can be categorized into physiological measurements such as measurements of heart rate [15-17] and behaviors, such as driver body movement [18-19] based on pressure distribution sensors, eye blinks [20-24], and facial expressions [25-29] based on camera sensors. Indirect driving-related measurements include measurements of pedal and steering activities [30-33] and reactions to specific events. The heart rate and the pulse wave [15-16] have been claimed to be effective physiological measures for evaluating driver drowsiness. Itoh et al. [18-19] claimed that driver body movement measurements with pressure distribution sensors can be used to detect driver drowsiness. When a driver becomes drowsy, he/she performs some activities in order to reduce his/her drowsiness [18]. Measurements of eyelid activities such as eye blinks, duration of closing eyelid, and eyelid closing velocity [20-24] are often used to evaluate driver drowsiness. The Karolinska sleepiness scale [26] is frequently used for subjective self-assessment. Kitajima et al. [27] proposed a sleepiness rating scale based on the facial expressions of the driver for external evaluation; this scale is often used for external evaluation in Japan. Ohsuga et al. [15] modified the Kitajima scale by considering the driver's resistance to sleepiness. Ishida et al. [28] suggested using facial muscular activities corresponding to a drowsy facial expression to estimate driver drowsiness automatically in real time. When considering the pedal and steering activities to evaluate driver drowsiness, steering delay [30-31], the lateral fluctuation of the vehicle [33], and standard deviation of the distance to a lead vehicle [18] are often used.

However, most of these methods tend to be unreliable. With physiological measures, ensuring correct judgment is difficult because of individual differences in physiological properties. Applications in the real world may also be impractical if they require restraints or invasive driver-monitoring equipment. 
Similarly, the evaluation of behavioral measures such as eye blinks and driver body movement is affected by individual differences. It is not easy to calibrate a decision criterion for driver drowsiness that is applicable to all drivers. Schemes based on drowsy facial expressions may not work effectively or properly if a driver wears a flu mask or sunglasses. The evaluation of steering activity for lane-keeping performance is affected by disturbances such as cross winds and road environment. Therefore, many researchers have attempted to develop a reliable approach for monitoring driver drowsiness based on a combination of direct driver-related and indirect driving-related measures [34-35].

A practical counter-measure to a driver falling asleep is to stop driving as soon as possible. Today, a common approach to preventing sleep-related vehicle accidents is that a system monitors the vehicle's lateral motion and steering activity, and alerts the driver and suggests taking a break when symptoms of sleepiness or fatigue are detected [36-38]. Warnings are expected to assist the driver's action selection. However, a driver who is aware of his/her drowsiness may still try to continue driving. In such a case, such warning-type support may fail to assure driver safety. This leads us to the question about what type of assistance system is necessary or effective for preventing sleep-related vehicle accidents.

In the vehicle safety control domain, some driver assistance systems have been already put into practical use [39-45]. Lane Departure Warning Systems (LDWS) monitor the vehicle's position with respect to the driving lane, and assist in maintaining safety by alerting drivers through visual, auditory, or haptic modalities [41-43] when the system anticipates a lane departure. However, in some situations, these systems may fail to prevent a lane departure. For example, when a driver's mental and physical functions are degraded or when a driver falls asleep, he/she cannot be expected to take the appropriate steering action. Humans may not always be able to cope with a given situation. On the other hand, Lane Keeping Assistance Systems (LKAS) that assist driver's action implementation by relieving their workload and maintaining the vehicle in the center of the lane also have been put into practical use [44-45]; however, the current LKAS do not take into account the characteristics of sleepy drivers and the driving context.

Against this background, we have proposed a driver assistance system with a dual control scheme, which tries to perform the safety control of a vehicle as well as identification of the driver's state (e.g., states of low arousal and degradation of mental and physical functions) simultaneously [46]. The assistance system works in a situation-adaptive manner [12], and it takes into account the characteristics of sleepy drivers and the driving context. We investigated the functionality and effectiveness of the driver assistance system for identifying driver drowsiness and preventing lane departure accidents.

In section II, a driver assistance algorithm with a dual control scheme is described. The design of the proposed assistance system that employs steering torque control based on a linear quadratic regulator is briefly presented based in [46]. In section III, a driving simulator experiment is described. We collected driver behavior data when the proposed assistance system was active. In section IV, the experimental results are presented. Driver state identification schemes are proposed, and the trade-off relationship between the accuracy and timeliness of drowsy driving detection is verified through a leave-one-out cross-validation (LOOCV) technique. Finally, in section V, the types of assistance system that are effective for preventing of sleep-related vehicle accidents are discussed.

\section{Driver Assistance with a DuAl CONTROL SCHEME}

In the field of control theory, the most adaptive controllers are based on the separation of parameter estimation and controller design [47]. Feldbaum [48] postulated two main properties that the control signals of optimal adaptive systems should have: (i) the system output cautiously tracks the desired reference value, and (ii) it excites the plant sufficiently for accelerating the parameter estimation process so that the control quality becomes better in future time intervals [47]. These properties are known as dual properties or dual features. Adaptive control systems with these two properties are named adaptive dual control systems [47]. Our driver assistance system is designed based on this main idea. In this study, the controlled object is a vehicle, and the object to be estimated through the control signal is the driver's state. Suppose a vehicle is going to deviate from a straight driving lane, as shown in Fig. 1.

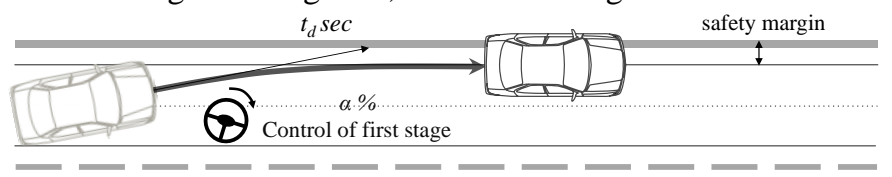

Fig. 1. Control of the first stage to prevent the lane departure.

When the assistance system anticipates that lane departure will occur in $t_{d}[s]$, the assistance system implements partial control $(\alpha \%)$ as control of the first stage to prevent lane departure from occurring. This control of the first stage is powerful enough to keep the vehicle in the lane, but it does not bring the vehicle back to the center of the lane. The vehicle ends up parallel to the lane markers at $0.5 \mathrm{~m}$ inside the lane markers. In other words, $(100-\alpha) \%$ of the steering control is left without being implemented. Normally, if a driver's situation awareness is appropriate, he/she will be uncomfortable in the event that the vehicle reaches near the lane boundary. If the driver performs the remaining control action within a time period of $t_{i}[s]$, as shown in Fig. 2, the assistance system judges that "he/she is paying attention to the traffic situation, and his/her understanding of the given situation is correct." However, the assistance system may implement the control of the first stage repeatedly during a short period of time if the driver is sleepy. In that case, the assistance system judges that "his/her situation awareness may be poor due to driver drowsiness," though he/she implements the proper steering action after the first-stage control.



Fig. 2. Driver's action to return to the center of the lane. 
On the other hand, if the driver does not perform the remaining control action within the time period of $t_{i}[s]$, the assistance system judges that "he/she is not paying attention to the traffic situation, and his/her understanding of the given situation is incorrect"; then the system will implement the remaining control as control of the second stage, as shown in Fig. 3. In addition, if the assistance system implements the second-stage control repeatedly, the assistance system judges that "the driver has lost situation awareness and cannot continue driving."

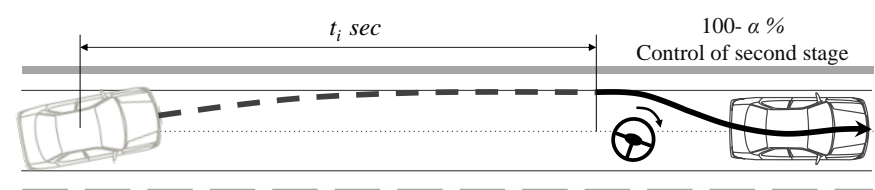

Fig. 3. Control of the second stage to return to the center of the driving lane.

Thus, the control of first stage has two purposes: (1) to effect the partial control initiated by the assistance system, preventing lane departure, and (2) enabling the assistance system to judge, through the interaction between the driver and the assistance system, whether the driver recognizes that the vehicle is going to deviate from the lane.

\section{A. Lane Departure Prediction}

Suppose a vehicle is going to deviate from the lane at a speed of $v(t)[\mathrm{m} / \mathrm{s}]$ and a deviation angle of $\psi(t)[\mathrm{rad}]$, as shown in Fig. 4, where $t$ is time. Judgment lines for lane departure are set at $0.5 \mathrm{~m}$ inside the lane markers on the right and left sides. The judgment line's position corresponds to the center of gravity of the vehicle in which the tire position strays into the adjacent lane. Lane departure is defined as the condition in which the lateral position $y(t)$ crosses the judgment line. Let $d(t)$ be the distance between $y(t)$ and the judgment line. The predicted value of the time margin, $t_{d}(t)$, to reach the judgment line is

$$
t_{d}(t)=d(t) / v_{y}(t)=d(t) /\{v(t) \cdot \sin \psi(t)\},
$$

where $v_{y}(t)$ is the lateral velocity. The assistance system initiates the control of the first stage at time $t^{*}$ when $t_{d}(t)$ becomes less than $1 \mathrm{~s}$. Determining the criterion for when to initiate the first-stage control is an important issue [49-50]. It is necessary to take into account two aspects: (1) to reduce the annoyance and (2) to maintain a sufficient time margin to avoid lane departure. It has been demonstrated that the time margin should be more than $0.9 \mathrm{~s}$ for avoiding the lane departure [51]. Thus, we established a criterion of $1 \mathrm{~s}$ with reference to previous research [51] for designing a lane-departure warning system.

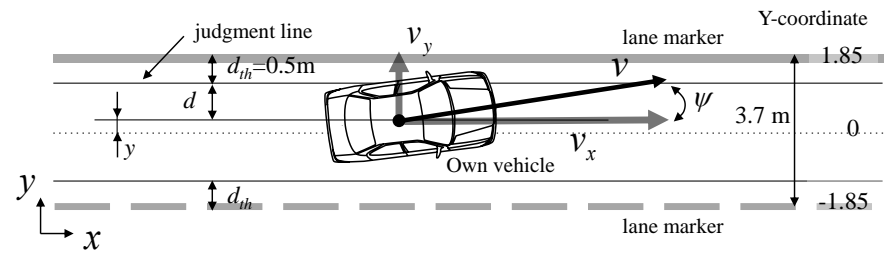

Fig. 4. Judgment of the lane departure.

\section{B. Safety Control}

Vehicle lateral control is classified into two types: (1) steering angle control, and (2) steering torque control [45, 52]. Steering angle control provides superior robustness because the non-linearity of the steering system is compensated in an inner loop in the steering angle servo algorithm [45]. However, the system does not allow steering intervention by the driver [45]. In contrast, steering torque control allows some steering intervention by the driver because it does not include the inner loop [45]. The proposed assistance system was designed using steering torque control to prioritize the driver's input torque when he/she takes the steering action.

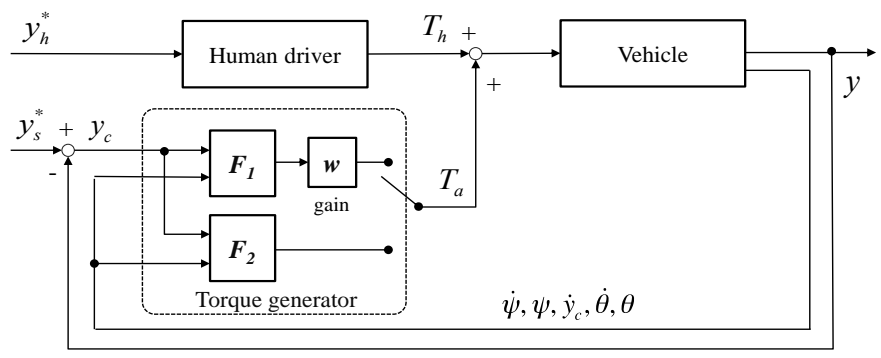

Fig. 5. Block diagram of the driver assistance system.

Fig. 5 shows the system's overall structure. Both the driver's input torque $T_{h}$ and the system's assistance torque $T_{a}(t)$ are applied to the steering wheel. The controller is designed using state feedback [46], where the state vector $x$ is composed of the yaw rate $\dot{\psi}$, yaw angle $\psi$, lateral velocity $\dot{y}_{c}$, lateral displacement $y_{c}$ between the system's target position $y_{s}{ }^{*}$ and the lateral position $y$, steering angle $\theta$, and steering angle velocity $\dot{\theta}$. The state vector $x$ is assumed to be measured or calculated using vehicle motion sensors. In addition, switching gains $F_{1}$ and $F_{2}$ are applied to the steering torque control [46], and the gain is switched according to the active stage of control (first or second). When $t_{d}(t)$ becomes less than $1 \mathrm{~s}$, the assistance system's target position $y_{s}{ }^{*}$ for the first-stage control is set as the judgment line, and the assistance system performs steering control so that the vehicle travels parallel to the lane markers at the judgment line. If no steering action by the driver is detected within $t_{i} \mathrm{~s}$, the assistance system's target position $y_{s}{ }^{*}$ is set as the center of the lane at time $t^{*}+t_{i}$, and the assistance system performs the second-stage control.

Based on the state feedback, the steering assistance torque $T_{a}(t)$ is calculated as follows:

$$
T_{a}(t)=\left\{\begin{array}{l}
-w F_{1} x(t), \quad t \in\left[t^{*}, t^{*}+t_{i}\right) \\
-F_{2} x(t), \quad t \in\left[t^{*}+t_{i}, t^{*}+t_{i}+5\right]
\end{array}\right.
$$

Assuming that the driver will take proper steering action within $5 \mathrm{~s}$ from the initiation of the first-stage control if his/her situation awareness is appropriate, we set time length $t_{i}$ to $5 \mathrm{~s}$. Furthermore, the assistance system completes the steering control $5 \mathrm{~s}$ after initiating the second-stage control.

In the proposed assistance system, an optimum control technique is applied to determine the assistance torque [46]. The state feedback gains $F_{1}$ and $F_{2}$ are designed so as to minimize 


$$
\begin{aligned}
& J_{j}=\int_{0}^{\infty}\left\{q_{y_{j}} y_{c}^{2}(t)+r_{j} T_{a}^{2}(t)\right\} d t, \quad j \in\{1,2\}, \\
& q_{y_{1}}=24.8, \quad q_{y_{2}}=1, \quad r_{1}=r_{2}=1,
\end{aligned}
$$

where $j$ represents the control state; $j=1$ represents the first-stage control, and $j=2$ represents the second-stage control. The coefficients $\left(q_{y_{j}}, r_{j}\right)$ were determined to obtain good control performance through numerical simulations using a general two-wheel model and a steering model [46].

\section{Detecting Driver's Action}

The assistance system monitors the driver's behavior over $5 \mathrm{~s}$ in addition to executing the first-stage control. If the driver takes steering action when the assistance system is implementing control of the first stage, the assisting torque must be reduced to minimize conflicts between the driver and the assistance system. As can be seen in Eqs. (2) and (5), a gain $w$ is introduced into the state feedback controller to reduce the assistance torque.

$$
w= \begin{cases}1 /\{1+0.001 \cdot \exp [-15 \cdot \psi(t)]\} & \text { if } \psi\left(t^{*}\right)>0 \\ 1 /\{1+0.001 \cdot \exp [15 \cdot \psi(t)]\} & \text { if } \psi\left(t^{*}\right)<0\end{cases}
$$

The gain $w$, which takes on values between 1 and 0 , is designed as a sigmoid function of the yaw angle, $\psi(t)$, to reduce the assisting torque smoothly; $w=1$ denotes full assistance, and $w=0$ denotes no assistance. As can be seen from $E q$. (5), as the direction in which the vehicle travel turns toward the center of the lane, the gain $w$ is reduced gradually from 1 to 0 . When the driver takes steering action to direct the vehicle to the center of the lane, the gain $w$ is reduced from 1 to 0 so as to produce a sigmoid curve, and the assistance torque $T_{a}$ is also gradually reduced. Further, if the driver takes unconscious or reflexive steering action to direct the vehicle towards the lane boundary, the assistance system provides torque to recover the target line for the first-stage control because the gain $w$ remains at 1 . Furthermore, when the gain $w$ is below 0.5 for $0.5 \mathrm{~s}$, the assistance system judges that "the driver took steering action to lead the vehicle back to the center of the lane," and then, cancels the control of the first stage. When the driver takes proper steering action, the controls by the assistance system and the driver are coupled. On the other hand, if the driver tries to take control during the second-stage control, the assistance system cancels the control of the second stage when the estimated input torque exceeds $3 \mathrm{Nm}$.

\section{DRIVING SIMULATOR EXPERIMENT}

We collected driver behavior data with the proposed assistance active in order to investigate the effectiveness of identifying driver drowsiness and preventing lane departure accidents. The experiment was conducted with the approval of the ethics committee of the Faculty of Engineering, Information and Systems at the University of Tsukuba.

\section{A. Apparatus}

The experiment was conducted in a fixed-base driving simulator equipped with the driver assistance system (Fig. 6).
The vehicle dynamics are calculated by a simulation computer manufactured by Mitsubishi Precision Co., Ltd., and the vehicle has an automatic gearbox. Three image-generating computers, connected to the simulation computer via a local-area network, generate the driving view. The view was shown to the driver using three projectors for the front and side screens. The field of views of the front and side screens was approximately 120 degrees. The driving simulator was equipped with a control-loaded steering system, a Moog-CL-R-E/HD/12Nm Multi Turn, which is controlled by a control-loading computer at a frequency of $2500 \mathrm{~Hz}$. The reaction torque as a self-aligning torque was simulated by Mitsubishi Precision software. In addition, a Smart Eye system was installed to measure the degree of opening of the eyelids. The Smart Eye system obtains eyelid opening, gaze, and head orientation information.

A $50 \mathrm{~km}$, two-lane, straight expressway was used in this experiment. The width of each lane was $3.7 \mathrm{~m}$. There were no buildings and other vehicles along the road (Fig. 6).

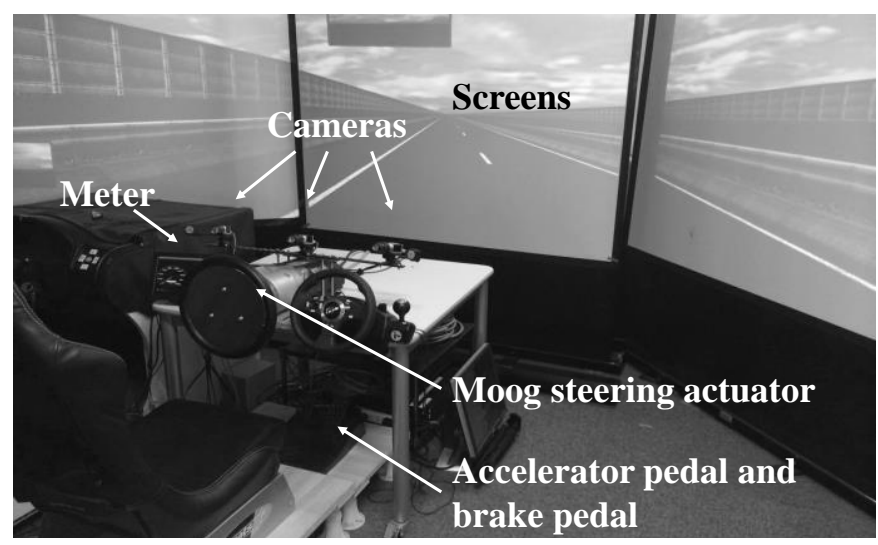

Fig. 6. A driving simulator equipped with the driver assistance system.

\section{B. Participants}

Twenty students (participants A-T, 15 males and 5 females) between the ages of 20-24 (mean: 22.0, s.d.: 1.19), who drive daily, participated. Each participant held a valid driver's license.

\section{Driving Tasks}

Participants were instructed to drive safely in the left-hand lane of a two-lane experimental course. The task imposed was to maintain the vehicle at the center of the lane. In this experiment, a speed regulator that maintains the speed of the vehicle automatically at $100 \mathrm{~km} / \mathrm{h}$ was used while participants were driving, in order to establish a monotonous driving environment and to induce drowsiness of the drivers. Thus, participants were required only to maintain lateral control of the vehicle. In addition, the participants were required to take proper steering action to lead the vehicle back to the center of the lane when they recognized the system's first-stage control for preventing the lane departure.

\section{Procedure}

The experiment was conducted in one day for each participant. First, each participant read and signed a written 
consent form, and informed consent was obtained from all participants. After receiving written instructions on the purpose, driving tasks, and procedures, the participants were informed that "the driver assistance system will support you in a lane departure situation by providing a torque on the steering wheel. The steering control is enough to keep the vehicle in the lane, but it does not bring the vehicle back to the center of the lane. If you recognize the partial control for preventing the lane departure, please implement the remaining steering action to return to the center of the lane."

Next, the participants completed practice drives to familiarize themselves with the simulator. In the training trials, the participants freely drove the driving simulator equipped with the proposed assistance system. Thus, all participants experienced the operation of the proposed driver assistance before the actual experiment.

In addition, the participants were asked to "please have a lunch between 12:00 to 12:50 if acceptable and avoid caffeine as much as possible" to induce their drowsiness. Trial driving was scheduled as follows: trial 1 was from 13:00 to 13:30; trial 2, from 14:00 to 14:30; and trial 3, from 15:00 to $15: 30$. Between the three trials, each participant took a 30-minutes break. The experiment took approximately $3.5 \mathrm{~h}$ per participant to complete.

\section{E. Sleepy Driver Behavior and Hypothesis}

Driving cannot simply be considered as a continuous closed-loop steering task to maintain the vehicle within a driving lane [53]. In the vehicle dynamics domain, preview-predictor models [54] as continuous closed-loop tasks based on visual feedback are often used to mimic the driver's action. Although drivers implement steering actions such as error-correcting mechanisms, they may pay attention to other aspects of the driving task, and drivers who are aware of their drowsiness may not be able to sufficiently maintain the continuous closed-loop task. Hence, steering delay [30-31] and lateral fluctuation [33] are often used to evaluate driver drowsiness.

In this study, we made the following hypothesis: If a participant falls asleep while driving, it is difficult for him/her to implement steering action to lead the vehicle to the center of the lane after the first-stage control. In addition, it is difficult for him/her to implement the required corrective steering for maintaining the vehicle at the center of the lane after the second-stage control. Therefore, when a participant falls asleep while driving, the system will implement both the first- and second-stage controls repeatedly.

\section{F. Measurements}

The following measurements were collected at a frequency of $120 \mathrm{~Hz}$ during the experiment: speed [km/h], lateral position $[\mathrm{m}]$, yaw angle [degrees], yaw rate $[\mathrm{rad} / \mathrm{s}]$, steering angle [degrees], gain $w$, driver's input torque [Nm], the system's assisting torque $[\mathrm{Nm}]$, the number of times the first- and the second-stage controls were initiated, and the degree of opening of eyelids [mm]. Facial images were collected at 30 frames per second. Moreover, the following data were calculated for each trial per participant in order to verify the hypothesis.

\section{1) Driver's Arousal State}

The driver's arousal state was evaluated by using both objective and subjective assessments in order to compensate for the ambiguity of the subjective method and for the low detection accuracy of eyelid opening.

- Number of the blinks [count]: The blinks were recorded automatically by the Smart Eye system. The measured blinks were counted every $20 \mathrm{~s}$.

- Sleepiness level based on facial expression: Facial expressions were evaluated based on the Kitajima scale [27] every $20 \mathrm{~s}$ by three evaluators at our laboratory; they evaluated the facial expressions of participants using the criteria listed in Table 1 in a subjective method. In this study, falling asleep implies level 5 of sleepiness. During the experiment, the evaluators could see only the participant's face through the recorded video. The sleepiness levels evaluated by the three evaluators were averaged and rounded for every rating. Prior to the experiment, the three evaluators practiced applying the subjective ratings in order to familiarize themselves with the process of external evaluation of recorded facial images.

TABLE I

SCALES OF SLEEPINESS LEVEL [27]

Level 1 Not Drowsy: Eye movement is rapid, and the time between blinks remains stable.

Level 2 Slightly Drowsy: Eye movement is slow.

Level 3 Moderately Drowsy: Blinks are slowly, the mouth moves, or the driver touches his/her face.

Level 4 Significantly Drowsy: Number of blinks increases noticeably, motions unnecessary for driving are observed, yawns are frequent, deep breathing is detected.

Level 5 Extremely Drowsy: Eyelids are almost closed, or the driver's head inclines to the front or rear.

\section{2) Lane-keeping Performance}

The following measures describe the lane-keeping accuracy with respect to the center of the driving lane.

- Standard deviation of the vehicle's lateral position [m]: It was calculated every $20 \mathrm{~s}$.

- Standard deviation of the steering wheel angle [degrees]: It was calculated every $20 \mathrm{~s}$.

- Mean and standard deviation of the absolute yaw angle [degrees]: The yaw angle was recorded when the assistance system anticipated that lane departure would occur within $1 \mathrm{~s}$.

\section{3) Safety Control Performance and Driver's Actions}

- Number of times the first- and second-stage controls were initiated [count]: They were counted per trial.

- Lateral position [m]: The relative frequency of the lateral position were calculated in the event when the assistance system was and was not actively controlling.

- Probable causes for the control of the first stage [\%]: 
Various causes may have led to a lane departure situation. For example, a participant's eyelids closed, or a participant was distracted. We evaluated the causes in a subjective method by using the facial image data with the Smart Eye system.

- The driver's steering action to return to the center of the driving lane: This measure describes whether or not the driver recognized that the vehicle was going to deviate from the lane by implementing the first-stage control. It was evaluated by using the facial image data.

- Time to implement the proper steering action [s]: It is a measure of the time length elapsed from initiation of the first-stage control to the time when the assistance system judged that "the participant implemented steering action to return to the center of the lane."

\section{G. Leave-one-out Cross-validation}

In the detection of drowsy driving, a trade-off must be made between the detection accuracy and the time for detection. If a system designer emphasizes the time to detect drowsy driving so that the driver is identified earlier, the assistance system's judgment may be incorrect or inappropriate. In contrast, if a system designer emphasizes the detection accuracy of drowsy driving to correctly identify the driver state, the assistance system may take a long time to identify the driver state. In detecting drowsy driving, there are four possible outcomes [50]: (a) correct detection, (b) false detection, (c) missed detection, and (d) correct rejection. False detection occurs when the assistance system determines that "the driver cannot continue driving," when in fact the driver can continue driving. On the other hand, missed detection occurs when the assistance system determines that "the driver can continue driving," when in fact the driver cannot continue driving. However, it is assumed that missed detection hardly occurs because it is unrealistic to expect a driver who has fallen asleep to take the proper steering action. Therefore, the detection criterion must balance the need for early detection with the need to avoid false detection.

In this study, driver state identification was based on the actions of the second-stage control system. The three different schemes chosen are further outlined in Section IV. D. These schemes were evaluated using a LOOCV technique with the measurement data of the participants for whom the second-stage control was implemented. The evaluation indices are as follows.

- The detection accuracy: The sleepiness level when the assistance system determined that "the driver cannot continue driving" was evaluated. The outcome was regarded as correct detection if the sleepiness level was at level 5, and as false detection if the sleepiness level was not at level 5. Additionally, the outcome was regarded as missed detection if the driver assistance system determined that "the driver can continue driving" owing to the driver's unconscious steering action when he/she had closed his/her eyelids. The analysis was performed with the recorded facial image data.

- The time to detect drowsy driving: The time elapsed before the assistance system determined that "the driver cannot continue driving" after the sleepiness level reached level 5 for the first time was evaluated. This evaluation was conducted by using the measurement data for the trials in which the participant fell asleep completely.

\section{RESUlts}

\section{A. Sleepiness Level and Lane-keeping Performance}

Even though individual differences were observed in terms of the tendency to be drowsy, 13 participants (A, C, D, E, G, I, $\mathrm{K}, \mathrm{N}, \mathrm{O}, \mathrm{P}, \mathrm{Q}, \mathrm{S}$, and T) were rated as "extremely drowsy (level 5 )" at least once. Of these, four participants (A, C, E, and S) fell asleep completely. In six out of 60 trials (20 participants $\times 3$ trials), a participant fell asleep completely.

Fig. 7 illustrates the relationships (a) between the sleepiness level based on facial expression and the standard deviation of the lateral position, (b) between the sleepiness level and the standard deviation of the steering wheel angle, and (c) between the sleepiness level and the number of blinks. Data from all participants data, which was calculated every $20 \mathrm{~s}$, was used in this analysis. A single-factor analysis of variance (ANOVA) with the sleepiness level on the standard deviation of the lateral position showed that the main effect of the sleepiness level was statistically significant $(\mathrm{F}(4,5335)=721.96, \mathrm{p}<0.01)$. Tukey's Honestly Significant Difference (HSD) test found statistically significant differences between levels 1 and 3,1 and 4, 1 and 5, between levels 2 and 3,2 and 4, 2 and 5, between levels 3 and 4, 3 and 5, and between levels 4 and 5 ( $p<0.01$ ).

A single-factor ANOVA with the sleepiness level on the standard deviation of the steering angle showed that the main effect of the sleepiness level was statistically significant $(\mathrm{F}(4$, $5335)=519.55, \mathrm{p}<0.01)$. Tukey's HSD test found statistically significant differences between levels 1 and 3,1 and 4, 1 and 5, between levels 2 and 3, 2 and 4, 2 and 5, between levels 3 and 4 , 3 and 5 , and between levels 4 and $5(\mathrm{p}<0.01)$.
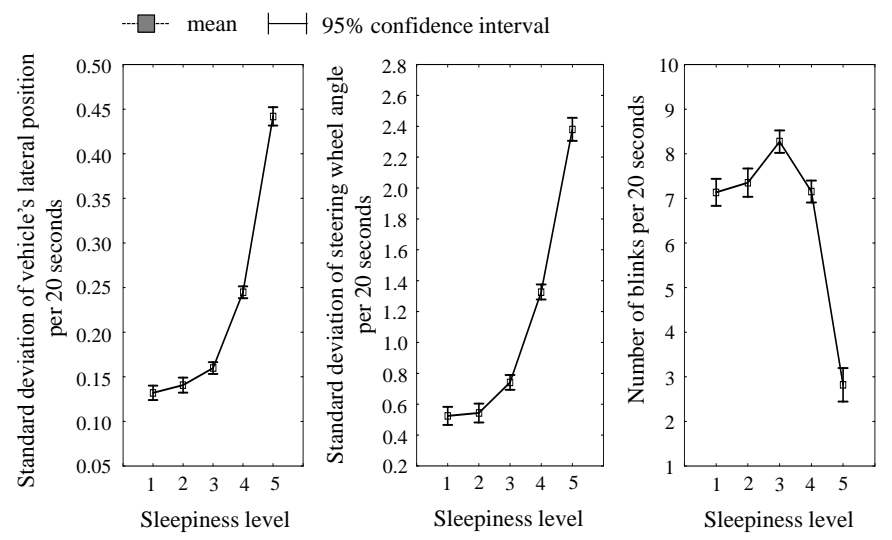

Fig. 7. Means and $95 \%$ confidence interval of the measures. Note that these results are affected by the system's first- and second-stage controls. 
A single-factor ANOVA with the sleepiness level on the number of blinks showed that the main effect of the sleepiness level was statistically significant $(\mathrm{F}(4,5068)=145.29$, $\mathrm{p}<$ 0.01). Tukey's HSD test found statistically significant differences between levels 1 and 3,1 and 5, between levels 2 and 3,2 and 5, between levels 3 and 4 , and 3 and 5, and between levels 4 and $5(\mathrm{p}<0.01)$.

For each measure, we performed a single-factor ANOVA with the five sleepiness levels. These results were consistent with those of previous studies on detecting driver drowsiness $[15,21-22,27]$. As the degree of sleepiness becomes larger, the driver's lane-keeping performance to maintain the vehicle at the center of the driving lane degraded. In general, the driver's corrective maneuvers to compensate for the lateral error between the target and actual vehicle positions are delayed when drivers feel sleepy, because visual occlusion often occurs as a result of longer eye blink durations [21-22]. The mean value of the absolute yaw angle was 0.91 degrees (s.d.: 0.37) when the assistance system anticipated that lane departure would occur within $1 \mathrm{~s}$. It is clear that the vehicle was going to deviate from the driving lane at very shallow angles.

\section{B. Safety Control Performances}

In this experiment, among the 709 times that the first-stage control was implemented, in 678 instances (95.6\%), the participant was at level 4 or 5 of sleepiness, as shown in Fig. 8. In addition, in 695 instances (98\%), the control was initiated when the driver's eyelids were going to be closed or were closed. In addition, among the 253 times that the second-stage control was implemented, 248 instances (98.4\%) occurred when the participant was at level 4 or 5 of sleepiness.

The vehicle did not deviate from the driving lane in any case in this experiment. Fig. 9 illustrates the relative frequency of the lateral position; the center of the lane is at $0 \mathrm{~m}$, lane marker positions are at -1.85 and $1.85 \mathrm{~m}$, and system's target line positions for the first-stage control are at -1.35 and $1.35 \mathrm{~m}$. Gray color denotes the lateral positions when the assistance system was not activated, and the black color denotes the lateral positions when the assistance system was activated. Fig. 9 shows clearly that the vehicle lateral position was maintained within the driving lane by the system's first-stage control.

The driver assistance system assisted the driver only when almost really needed in lane departure situations, as shown in Fig. 10. Fig. 10 illustrates data for $70 \mathrm{~s}$ of the time-series data over the interval [ $470 \mathrm{~s}, 540 \mathrm{~s}$ ] during trial 3 of participant $\mathrm{E}$ in which the sleepiness level of the participant was at level 5 (extremely drowsy). It shows the states of the assistance system [1; implementing the first-stage control, 2; implementing the second-stage control], lateral position [m], assisting torque [Nm], driver's input torque [Nm], eyelid opening [mm], and sleepiness level. Approximately $5 \mathrm{~mm}$ of eyelid data denotes that the driver's eyelids were closed. In $70 \mathrm{~s}$, the participant's eyelids were almost closed, and the lateral fluctuation of the vehicle tended to become bigger. The assistance system prevented lane departure thrice during the $70 \mathrm{~s}$ episode. We confirmed that the participant opened his/her eyelids that had been closed after the system's first-stage control and provided



Fig. 8. Number of the first- and second-stage controls.

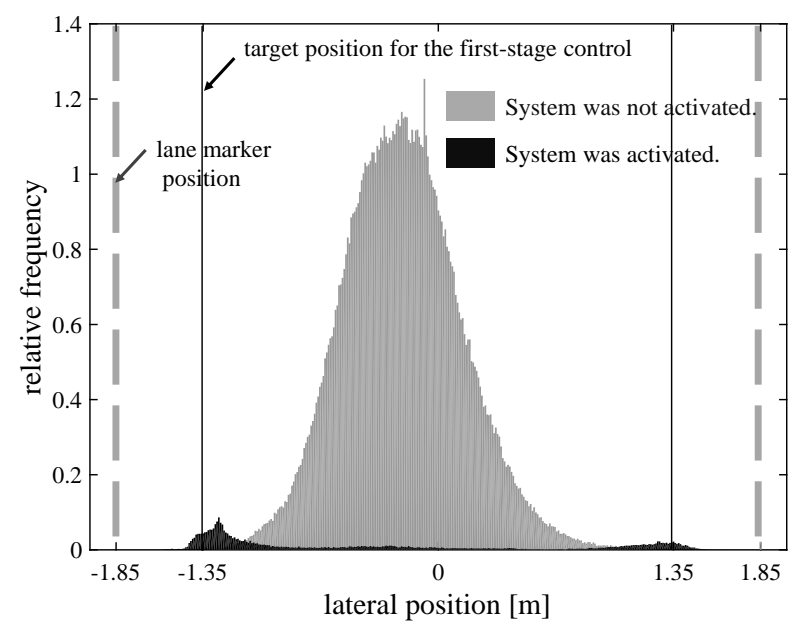

Fig. 9. Relative frequency of the lateral position. The mean value was $-0.20 \mathrm{~m}$. The driver's seat was installed at the center of the front screen. This is the result of participants trying to maintain their seat position at the center of the lane.

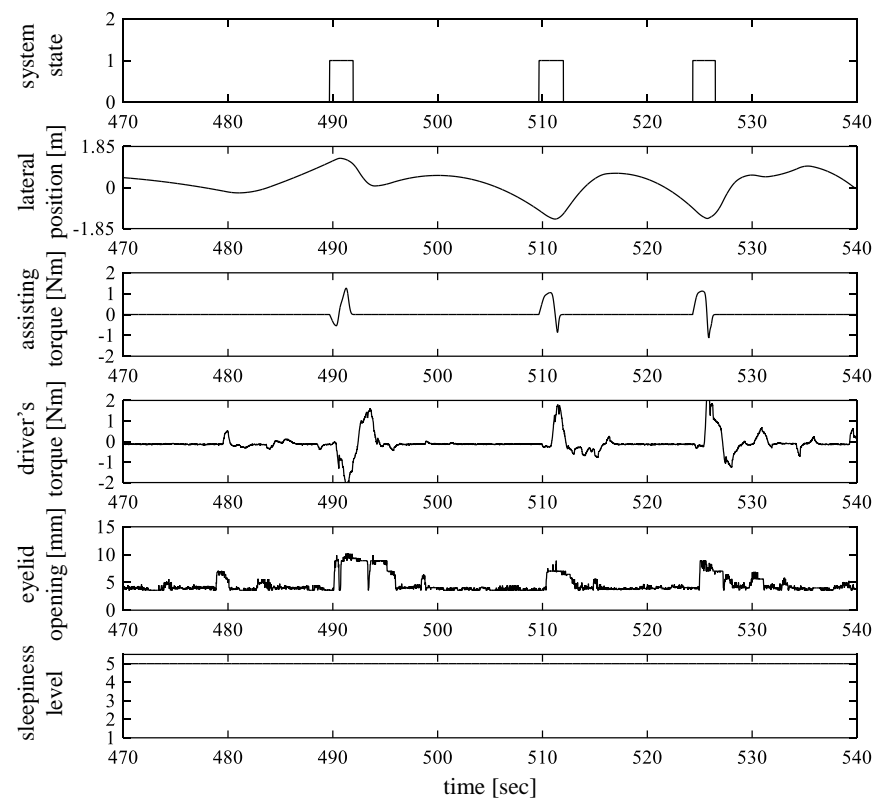

Fig. 10. Time-series data of 70 seconds during trial 3 of participant $E$.

steering torque to return to the center of the lane. Thus, the vehicle returned to the center of the lane by both the assistance system's control and the driver's steering action. 


\section{Sleepiness Level and Driver's Actions}

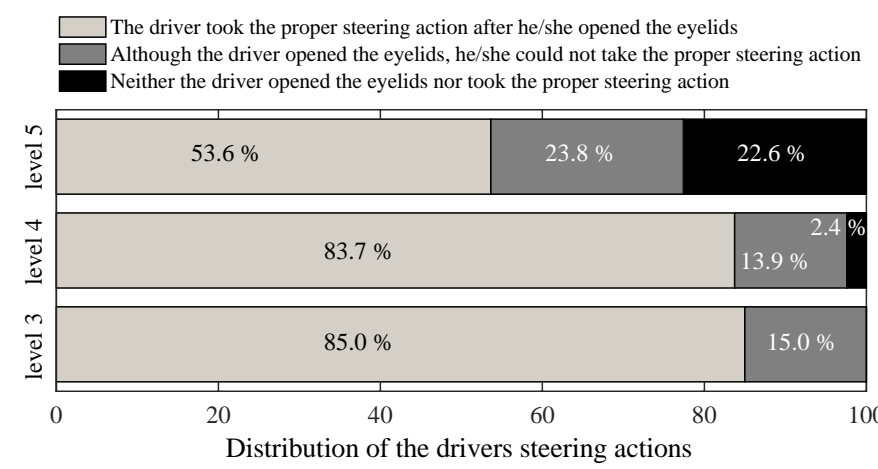

Fig. 11. Distribution of driver's actions. to the center of the lane if he/she falls asleep. Fig. 13 is a typical

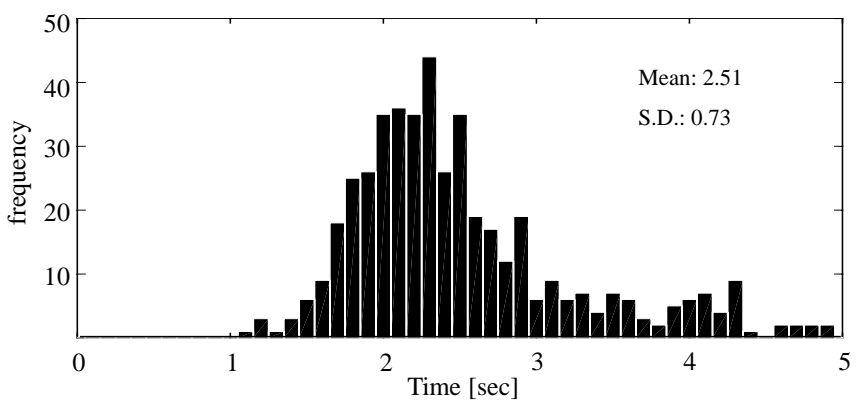

Fig. 12. The time elapsed before the driver took the proper steering action after the first-stage control was initiated. There are no data where the elapsed time is over 5 seconds because the assistance system implements the second-stage control at time of $t^{*}+5$ seconds.

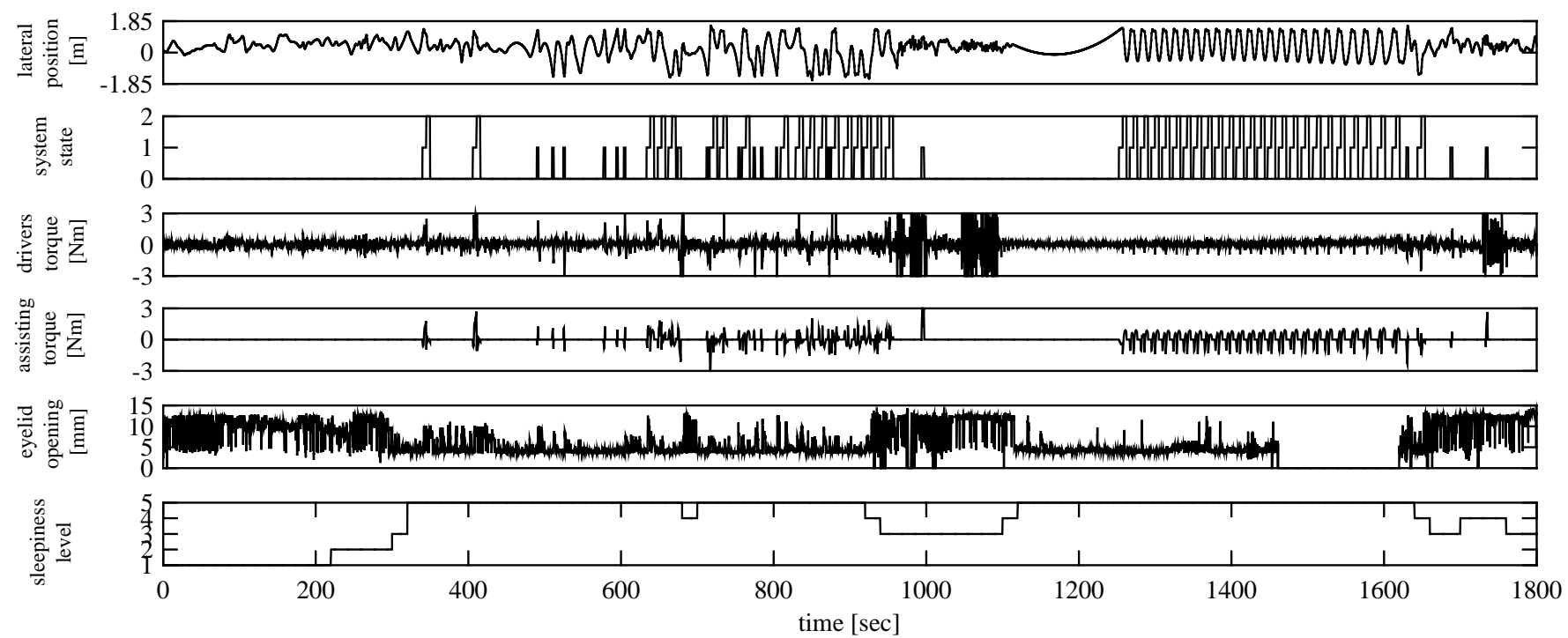

Fig. 13. Time-series data of 1800 seconds during trial 1 of participant E.

After the assistance system initiated the first-stage control, three types of driver action were observed: (a) the driver took the proper steering action after he/she opened his/her eyelids, (b) although the driver opened his/her eyelids, he/she could not take the proper steering action, and (c) the driver neither opened his/her eyelids nor took the proper steering action. Fig. 11 shows the distribution of driver's actions after the first-stage control. When the sleepiness of participants was at level 3, they could take the proper steering action in $85 \%$ of the cases to lead the vehicle to the center of the lane after the first-stage control. Fig. 12 illustrates a histogram (mean: 2.51, s.d.: 0.73) of the time elapsed before the driver took the proper steering action after the assistance system initiated the control of the first stage. This result was analyzed by using type (a) data. It clear from Fig. 12 that the driver can recognize the first-stage control and can take the proper steering action within $5 \mathrm{~s}$ if his/her situation awareness is appropriate.

On the other hand, when the participant was at level 5 of sleepiness, type (c) occurred in $22.6 \%$ of the cases, and proper steering action after the first-stage control was not taken in $46.4 \%$ of the cases. These results suggest that it is difficult for a participant to implement steering action that returns the vehicle example of the results in which the assistance system implements both the first- and second-stage controls repeatedly when the participant falls asleep while driving, and it illustrates the time-series data for $1800 \mathrm{~s}$ during trial 1 for participant E. As can be noticed from the eyelid opening data, we can confirm that the driver's eyelids closed at approximately $300 \mathrm{~s}$, and the sleepiness level gradually rose from level 2 to level 5 . The state of the assistance system shows that it implemented the controls of the first and the second stages repeatedly after the sleepiness level of the driver reached level 5. This trend was common among participants (A, C, E, and S) who fell asleep completely. Further, the opening of the eyelids of the participant returned to a normal state (approximately $13 \mathrm{~mm}$ ) at approximately $920 \mathrm{~s}$. In the $150 \mathrm{~s}$ for the interval $[950 \mathrm{~s}, 1100 \mathrm{~s}$ ], the participant frequently rotated the steering wheel in the left and right directions, as shown in the driver's input torque data. This is because the participant was making an effort to wake up or to maintain his/her arousal level. However, we can confirm that the eyelids closed at approximately $1110 \mathrm{~s}$, and the participant fell asleep again. As can be noticed from the state of the assistance system and the lateral position data, participant E, who fell asleep, continued driving from at approximately 1110 
$\mathrm{s}$ until at approximately $1620 \mathrm{~s}$, and the assistance system implemented the first- and second-stage controls repeatedly.

\section{Accuracy and Timeliness of Drowsy Driving Detection}

We performed the LOOCV with the measurement data of the 14 participants for whom the second-stage control was implemented. Let us consider the measurement data of participant $\mathrm{A}$ as the test data. Then, the measurement data of the remaining 13 participants are the training data. The time intervals between two consecutive controls of the second stage were analyzed with the measurement data of 13 participants, excluding participant A. Fig. 14 shows the time intervals between two consecutive controls when the sleepiness level of the participant was at level 4 or 5 .
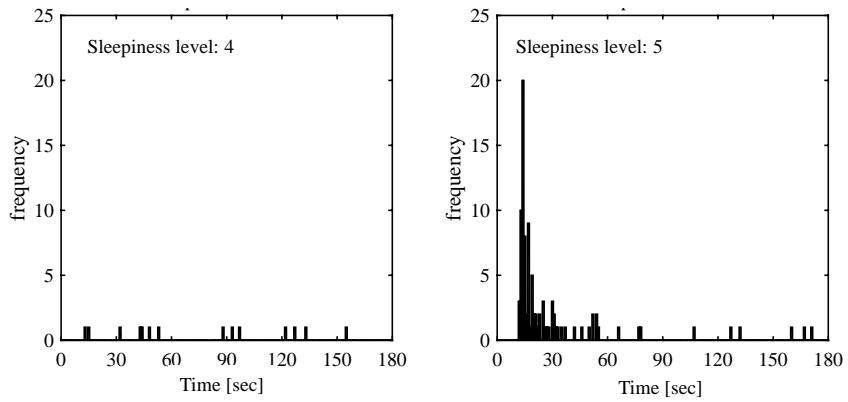

Fig. 14. Time interval between the two consecutive controls.

In many cases, the time interval between two consecutive controls was within $30 \mathrm{~s}$ when the participant was at a sleepiness level of 5 . The time length $t_{e}$, which is the time at which the cumulative distribution reached $70 \%$, was used as an index for constructing the driver state identification scheme. When the measurement data of participant A were the test data, the time length $t_{e}$ was $29.2 \mathrm{~s}$. We propose three schemes to evaluate the accuracy and the timeliness of drowsy driving detection:

Scheme 1: The assistance system judges that the driver cannot continue driving when the second-stage control is implemented for the first time.

Scheme 2: The assistance system judges that the driver cannot continue driving when the second-stage control is implemented twice within time $t_{e}[\mathrm{~s}]$.

Scheme 3: The assistance system judges that the driver cannot continue driving when the second-stage control is implemented thrice within time $2 t_{e}$ [s].

The LOOCV was repeated so that all measurement data were treated as test data.

\section{1) Detection Accuracy}

In the LOOCV, the time interval $t_{e}$ was less than $30 \mathrm{~s}$ in all cases. Fig. 15 shows the distribution of the sleepiness level at the time when the assistance system judged that "the driver cannot continue driving" in each scheme. The rate of false detection was $73 \%$ when adopting scheme $1,12 \%$ when adopting scheme 2 , and $0 \%$ when adopting scheme 3 . Therefore, correct detection was achieved in $88 \%$ of the cases for scheme 2 , and in $100 \%$ of the cases for scheme 3 . Furthermore, no case of missed detection occurred because an event in which the driver took unconscious steering action after the first-stage control



Fig. 15. Distribution of the sleepiness level.
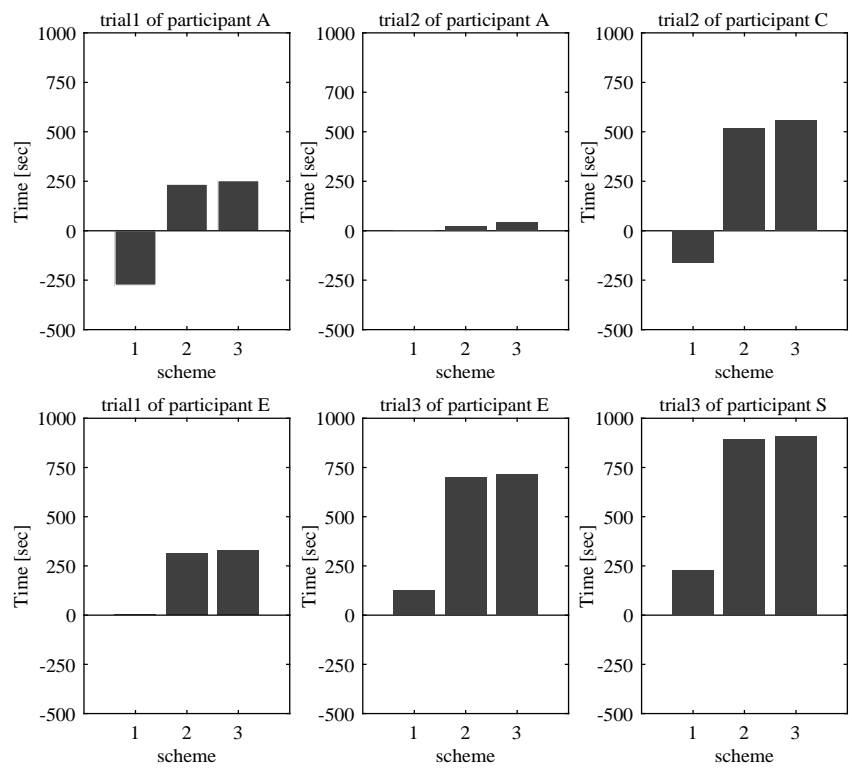

Fig. 16. Time elapsed before the assistance system judged that "the driver cannot continue driving" after the sleepiness level reached level 5 .

was not observed.

\section{2) Time to Detect Drowsy Driving}

Fig. 16 shows the time elapsed before the assistance system judged that "the driver cannot continue driving" after the sleepiness level reached level 5 for the first time. The six trials correspond to those in which the participant fell asleep. The time elapsed before the assistance system determined that "the driver cannot continue driving" differed according to the driver's state because he/she made an effort to wake up or to maintain his/her arousal level. Schemes 3 and 2 required more time to identify the driver state than scheme 1 . However, the difference in the time elapsed between schemes 3 and 2 was $20.8 \mathrm{~s}$ on average.

\section{Discussions}

\section{A. Effectiveness of the Dual Control Scheme Based Assistance}

In this experiment, the lane-keeping performance degraded as the degree of sleepiness rose from level 2 to level 5. In particular, when a participant was at a sleepiness level of 4 or 5 , the standard deviations of the steering wheel angle and the vehicle's lateral position tended to become larger. Longer eye blink duration, which causes visual occlusion, was observed during the experiment. We confirmed that the vehicle would 
deviate from the driving lane at very shallow angles because the participant's corrective maneuver was delayed during the visual occlusion.

In the lane departure situation, the proposed driver assistance system implemented the minimum-required partial control. The assistance system extended the time to lane-crossing and gave the driver a chance to voluntarily take the action needed. When the driver failed to implement the steering action needed within a limited time, the assistance system determined that "he/she is not paying attention to the traffic situation and his/her understanding of the given situation is incorrect" and executed the remaining control on behalf of the driver. The driver assistance system assisted participants only when the need was imminent, such as when his/her eyelids were closed. The vehicle did not deviate from the lane in any case. The driver assistance system was effective for preventing lane departure accidents. The experimental results also showed that the assistance system implemented both the first- and second- stage controls repeatedly when the participant was at a sleepiness level of 5. It is clear that it is difficult for a participant to take proper steering action after the system's first-stage control and to implement corrective steering to remain within the lane after the system's second-stage control if he/she falls asleep. These results support the hypothesis that we made in Section III.

When the assistance system implemented the control of the second stage repeatedly, it judged that "the driver has lost situation awareness and cannot continue driving." If a driver's mental and physical states are degraded or a driver falls asleep, the assistance system should lead to stop the vehicle as soon as possible. The schemes for drowsy-driving detection were proposed, and the trade-off relationship between the accuracy and timeliness of drowsy-driving detection was verified through the LOOCV technique. Correct detection was achieved in $88 \%$ of the cases for scheme 2 , and in $100 \%$ of the cases for scheme 3 . The results suggest that scheme 3 or 2 can be useful for detecting drowsy driving. We demonstrated that a driver assistance system based on scheme 3 or 2 can balance the need for early detection with the need to avoid false detection.

However, we also observed situations in which a sleepy driver continues driving with the assistance system for preventing lane departure. This situation is not desirable. The driver should stop driving as soon as possible. We propose a multi-layered assistance via the dual control scheme. The driver is assumed to be in command. However, the driver may not always be able to cope with the given situation due to low arousal and degradation of mental and physical functions. Therefore, (a) when the assistance system initiates the first-stage control for the third time, the assistance system gives alerts the driver and suggests that taking a break, and (b) when the assistance system initiates the second-stage control for the second time within $30 \mathrm{~s}$, the assistance system determines that "the driver cannot continue driving", and thus it decelerates the vehicle and applies lane centering control to slowly stop the vehicle. If the driver engages the accelerator while the control for stopping the vehicle is underway, the control to slowly stop the vehicle is canceled. Fig. 17 illustrates the circumstance of a sleep-related accident in which an overnight bus collided with a parked vehicle on the Hokuriku Expressway in Japan. Even though the overnight bus ran into guardrails on the left and right sides of the road, the commercial driver did not take action to avoid the accident. The experimental results suggest that the proposed driver assistance system can be effective in such a case for identifying driver drowsiness and preventing lane departure accidents. The system's target line that is set when the assistance system anticipates lane departure represents a virtual sticky-guardrail in a metaphor. Even though the vehicle is in contact with the virtual sticky-guardrail several times, if the driver does not take steering action to lead the vehicle to the center of the lane, the assistance system determines that "the driver cannot continue driving". The proposed assistance system can be commercially used in general cars equipped with electric power steering and a device to recognize with respect to white lines on roads, and can be realized by monitoring only vehicle information.

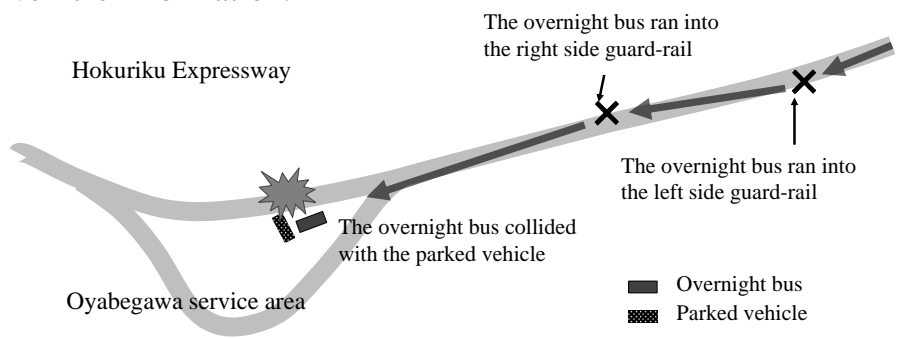

Fig. 17. Sleep-related vehicle accident on Hokuriku Expressway in Japan.

\section{B. Limitations and Future Work}

It is necessary to improve the driver state identification algorithm to be able to identify the driver state earlier. This study focused on designing the human machine interaction through the steering wheel. Monitoring accelerator pedal and/or brake pedal actions may be effective for identifying the driver state. This work also assumed that the road is straight, but the possibility of road departure crashes may be high along curves. Thus, it is necessary to improve the control algorithm for applying it to more complex situations. In addition, this study does not take into account lane change behavior and evasive maneuvers in an actual driving scene. A system design that can respond to these situations is required.

There are various ways for bringing a vehicle to a stop after the assistance system determines that "the driver cannot continue driving." One way is to stop the vehicle in the driving lane in which the vehicle is moving. The other is to pull over and to stop on the side of the road. In the latter case, a lane change may be required to stop the vehicle on the side of the road. Then, it will be necessary to notify the surrounding traffic to the emergency stop. Those conducting future research should investigate the best manner for stopping the vehicle while ensuring vehicle safety.

In our experiment, 20 students between the ages of 20-24 who drive daily participated. We recognize the importance of studying to a wide range of age groups. In general, traffic accidents caused by elderly drivers have been increasing owing to the degradation in physical ability in terms of perception, recognition, decision-making, and action implementation. Research covering a wide range of age groups is still lacking. In addition, in our study, participants were required to maintain only lateral control of the vehicle in order to induce driver 
drowsiness. In real-world driving, the driver manages the speed while making an effort to wake up when he/she feels sleepy. There is a need to conduct a study in an environment that allows the speed to be adapted to the driver. Research is also limited by the limitation of the simulator environment because the generated stimuli and/or sensation of risk are usually low as compared with a real driving environment. These limitations could be eliminated in the future to use actual experimental vehicles in a real driving environment. Further studies are necessary to investigate whether drivers accept the assistance system's behavior in a more realistic driving context when they do not have prior experience of interacting with the assistance system.

\section{CONCLUSIONS}

This paper discussed an assistance system that is effective for preventing of sleep-related vehicle accidents. A multi-layered assistance with a dual control scheme, which could assist in reducing sleep-related accidents, was presented. The assistance system judges the driver's state in a multi-layered way through the interaction between the driver and the assistance system in addition to executing the first- and second-stage controls to maintain safety.

Through a driving simulator experiment, we investigated the system's functionality and the effectiveness with which it could identify driver drowsiness and prevent lane departure accidents. The assistance system assisted the driver only when almost really needed in lane departure situations. We made the hypothesis that the driver cannot implement the action needed to maintain safety when he/she falls asleep, and in such an event, the assistance system will implement the safety control repeatedly; the results showed that the assistance system implemented both the first- and second-stage controls repeatedly when a participant fell asleep. Thus, the results supported our hypothesis. Driver state identification schemes based on the actions of the second-stage control were proposed, and the trade-off between detection accuracy and the time to detect drowsy driving was investigated through LOOCV analysis. We found that in many cases, the time interval between two consecutive controls of the second stage was within $30 \mathrm{~s}$ when a participant was at level 5 of sleepiness. Based on the results of the LOOCV, we conclude that the assistance system can be useful for detecting drowsy driving when adopting the following scheme: the assistance system judges that "the driver cannot continue driving" when the second-stage control is initiated twice within $30 \mathrm{~s}$ or thrice within $60 \mathrm{~s}$. Although more research is needed, the experimental results demonstrate that the proposed assistance system can contribute to identifying driver drowsiness and preventing lane departure accidents.

\section{REFERENCES}

[1] National Highway Traffic Safety Administration, "Traffic safety facts: drowsy driving," Washington, DC: United States Department of Transportation, Rep. DOT HS 811 449, 2011.

[2] A. I. Pack, A. M. Pack, E. Rodgman, A. Cucchiara and D. F. Dinges, "Characteristics of crashes attributed to the driver having fallen asleep," Accident Anal. \& Prevention, vol. 6, pp. 769-775, 1995.

[3] P. H. Langlois, M. H. Smolensky, B.P. Hsi and F. W. Weir, "Temporal patterns of reported single-vehicle car and truck accidents in Texas,
U.S.A. during 1980-1983," Chronobiology Int., vol. 2, no. 2, pp. 131-140, 1985.

[4] M. M. Mitler and J.C. Miller, "Methods of testing for sleepiness," J. of Behavioral Medicine, vol. 21, no. 4, pp. 171-183, 1996.

[5] J. A. Horne and L. A. Reyner, "Sleep related vehicle accidents," Brit. Med. J., vol. 6979, pp. 565-567, 1995.

[6] G. Maycock, "Sleepiness and driving: the experience of U.K. car drivers," Accident Anal. \& Prevention, vol. 29, no. 4, pp. 453-462, 1997.

[7] P. Philip, I. Ghorayeb, R. Stoohs, J. C. Menny, P. Dabadie, B. Bioulac and C. Guilleminault, "Determinants of sleepiness in automobile drivers," $J$. of Psychosomatic Res., vol. 4, no. 3, pp. 279-288, 1996.

[8] S. Barbarino, L. Nobili, M. Beelke, F. D. C. Phy and F. Ferrillo, "The contributing role of sleepiness in highway vehicle accidents," Sleep, vol. 24, pp. 203-206, 2001.

[9] H. Summala and T. Mikkola, "Fatal accidents among car and truck drivers: effects of fatigue, age, and alcohol consumption," Hum. Factors, vol. 36, no. 2, pp. 315-226, 1994.

[10] J. Horne and L. Reyner, "Vehicle accidents related to sleep: a review," Occupational and Environmental Medicine, vol. 56, pp. 289-294, 1999.

[11] R. Parasuraman, T. B. Sheridan and C. D. Wickens, "A model for types and levels of human interaction with automation," IEEE Trans. of Syst., Man, and Cybern., vol. 30, no. 3, pp. 286-297, 2000.

[12] T. Inagaki, "Design of human-machine interactions in light of domain-dependence of human-centered automation," Cogn., Technol. \& Work, vol. 8, no. 3, pp. 161-167, 2006.

[13] T. Inagaki, "Smart collaborations between humans and machines with mutual understanding," Annu. Rev. in Control, vol. 32, pp. 253-261, 2008.

[14] HAVEit (Highly Automated Vehicles for Intelligent Transport) Project, "The future of driving," Deliverable D61.1 Final Rep., 2011

[15] M. Ohsuga, Y. Kamakura, Y. Inoue, Y. Noguchi, K. Shimada and M. Mishiro, "Estimation of driver's arousal state using multi-dimensional physiological indices," Eng. Psychology and Cogn. Ergonom., Lecture Notes in Computer Science, vol. 6781, pp. 176-185, 2011.

[16] E. Fujita, Y. Ogura, N. Ochiai, T. Miao, T. Shimizu, K. Murata, T. Kamei, Y. Ueno and S. Kaneko, "Experimental study on prediction of sleep during wakefulness," in Proc. First Int. Symp. on Advanced Technol. of Vibration and Sound, Hiroshim, Japan, pp. 282-287, 2005

[17] G. Keckluindand and T. Åkerstedt, "Sleepiness in long distance truck driving: an ambulatory EEG study of night driving," Ergonom., vol. 36, no. 9, pp. 1007-1017, 1993.

[18] M. Itoh, R. Ishikawa and T. Inagaki, "Evaluating body movement of a drowsy driver with pressure distribution sensors," in Proc. 3rd Int. Symp. on Future Active Safety Technology Towards Zero Traffic Accident, Gothenburg, Sweden, pp. 267-272, 2015.

[19] M. Itoh, H. Nagasaku and T. Inagaki, "Analyses of driver's body movement for detection of hypovigilance due to non-driving cognitive task," in Proc. IFAC-CTS, Delft, Netherlands, pp. 644-648, 2006.

[20] T. Åkerstedt, M. Ingre, G. Kecklund, A. Anund, D. Sandberg, M. Wahde, P. Philip and P. Kronberg, "Reaction of sleepiness indicators to partial sleep deprivation, time of day and time on task in a driving simulator -the DROWSI Project," J. of Sleep Res., vol.19, no. 2, pp. 298-309, 2010.

[21] M. Ingre, T. ÅKerstedt, B. Peters, A. Anund and G. Kecklund, "Subjective sleepiness, simulated driving performance and blink duration: examining individual differences," J. of Sleep Res., vol. 15, no. 1, pp. 47-63, 2006.

[22] D. Sandberg, "The performance of driver sleepiness indicators as a function of interval length," in Proc. 14th Int. IEEE Annu. Conf. on Intell. Transp. Syst., Washington, DC, USA, pp. 1735-1740, 2011.

[23] I. G. Damousis and D. Tzovaras, "Fuzzy fusion of eyelid activity indicators for hypovigilance-related accident prediction," IEEE Trans. on Intell. Transp. Syst., vol. 9, no. 3, 2008.

[24] S. Hu and G. Zheng, "Driver drowsiness detection with eyelid-related parameters by support vector machine," Expert Syst. with Appl., vol. 36, no. $4,2009$.

[25] T. Åkerstedt and M. Gilberg, "Subjective and objective sleepiness in the active individual," Int. J. of Neuroscience, vol. 52, no. 1, pp. 29-37, 1990.

[26] K. Kaida, M. Takahashi, T. Åkerstedt, A. Nakata, Y. Otsuka, T. Haratani and K. Fukusawa, "Validation of the karolinska sleepiness scale against performance and EEG variables," Clinical Neurophysiology, vol. 117, no. 7, pp. 1574-1581, 2006.

[27] H. Kitajima, N. Numata, K. Yamamoto and Y. Goi, "Prediction of automobile driver sleepiness (1st report)," J. of the Jan. Soc. of Mech. Eng., Vol. 63, No. 613, pp. 3059-3066 in Japanese, 1997. 
[28] K. Ishida, A. Ichimura and M. Kamijo, "A study of facial muscular activities in drowsy expression," Int. J. of Kansei Eng., vol. 9, no. 2, pp. $57-66,2010$.

[29] S. Hachisuka, K. Ishida, E. Takeshi and K. Masayoshi, "Facial expression measurement for detecting driver drowsiness," Eng. Psychology and Cogn. Ergonom., Lecture Notes in Computer Science, vol. 6781, pp. 135-144, 2011.

[30] W. A. McDonald and E. R. Hoffman, "Review of relationships between steering wheel reversal rate and driving task demand," Hum. Factors, vol. 22, no. 6, pp. 733-739, 1980.

[31] J. Krajewski, D. Sommer, U. Trutschel, D. Edwards and M. Golz, "Steering wheel behavior based estimation of fatigue," in Proc. 5th Int. Driving Symp. on Hum. Factors in Driver Assessment and Design, Salt Lake, Utah, USA, pp. 118-124, 2009.

[32] M. Farid, N. Kopf, H. Bubb and A. Essaili, "Methods to develop a driver observation system used in an active safety system," SafetyLit, vol. 1960, pp. 639-5650, 2006.

[33] W. W. Wierwille and L. A. Ellsworth, "Evaluation of driver drowsiness by trained raters," Accident Anal. and Prevention, vol. 26, no. 5, pp. 571-581, 1994.

[34] M. Omidyeganeh, A. Javadtalab and S. Shirmohammadi, "Intelligent driver drowsiness detection through fusion of yawning and eye closure," in Proc. IEEE Int. Conf. on Virtual Environments Human-Computer Interfaces and Measurement Systems, Ottawa, Canada, 6pages, 2011.

[35] I. G. Daza, N. Hernandez, L. M. Bergasa, I. Parra, J. J. Yebes, M. Gavilan, R. Quintero, D. F. Llorca and M. A. Sotelo, "Drowsiness monitoring based on driver and driving data fusion," in Proc. 14th Int. IEEE Conf. on Intell. Transp. Syst., Washington, DC, USA, pp. 1199-1204, 2011.

[36] Volvo Car Corporation, "Volvo cars introduces new systems for alerting tired and unconcentrated drivers", Press information, August 28, 2007

[37] Daimler AG, "Method and computer program for identification of inattentiveness by the driver of a vehicle", U.S. Patent 7283056, December 24, 2004.

[38] Volkswagen, "Device for fatigue warning in motor vehicles having a run-up alarm system", U.S. Patent 7821384, October 26, 2010.

[39] Fuji Heavy Industries Ltd., "Warning system for vehicle", U.S. Patent 5699057, December 16, 1997.

[40] S. Motoyama, T. Ohta, T. Watanabe and Y. Ito, "Development of lane departure warning system," in Proc. 7th Intelli. Transp. Syst. World Congr., Torino, Italy, 8pages, 2000.

[41] K. Suzuki and H. Jansson, "An analysis of driver's steering behavior during auditory or haptic warnings for the designing of lane departure warning system," JSAE Rev., vol. 24, no. 1, pp. 65-70, 2003.

[42] J. Navarro, F. Mars and JM. Hoc, "Lateral control assistance for car drivers: a comparison of motor priming and warning systems", Hum. Factors, vol. 49, no. 5, pp. 950-60, 2007.

[43] M. Deroo, JM. Hoc and F. Mars, "Influence of risk expectation on haptically cued corrective manoeuvres during near lane departure," Ergonom., vol. 55, no. 4, pp. 465-475, 2012.

[44] Nissan Motor Co., Ltd., "Lane keeping assist device and lane keeping assist method," EP Patent 2248710, November 10, 2010.

[45] M. Shimakage, S. Satoh, K. Uenuma and H. Mouri, "Design of lane-keeping control with steering torque input," JSAE Rev., vol. 23, no. 3, pp. 317-323, 2002.

[46] Y. Saito, M. Itoh and T. Inagaki, "Dual control theoretic driver assistance -Dynamic characteristics of steering torque control based on linear quadratic regulator," in Proc. 2014 IEEE Int. Conf. on Syst., Man and Cybern., Sandiego, USA, pp. 1738-1743, 2014.

[47] N. Filatov and H. Unbehauen, "Adaptive dual control: theory and applications," Springer, 2014.

[48] A. A. Feldbaum, "Theory of dual control (I)," Automation and Remote Control, vol. 21, no. 9, pp. 1240-1249, 1960.

[49] K. Lee and H. Peng, "Evaluation of automotive forward collision warning and collision avoidance algorithms," Vehicle System Dynamics, vol. 43, no. 10 , pp. $735-751,2005$.
[50] R. Parasuraman, P. A. Hancock and O. Olofinboba, "Alarm effectiveness in driver-centerd collision-warning systems," Ergonom., vol. 40, pp. 390-399, 1997.

[51] K. Suzuki, T. Wakasugi and H. Soma, "Designing method of warning timing based on the time criterion for lane departure warning system," Trans. of the Soc. of Instrument and Control Engineers, vol. 38, no. 6, pp. 567-573, 2002.

[52] D. A. Abbink, M. Mulder and E. R. Boer, "Haptic shared control: smoothly shifting control authority?," Cogn., Technol., \& Work, vol. 14, no. 1, pp. 19-28, 2012.

[53] H. Godthelp, P. Milgram and G. Blaauw, "The development of a time-related measure to describe driving strategy," Hum. Factors, vol. 26, no. 3, pp. 257-268, 1984.

[54] K. Yoshimoto, "Simulation of man-automobile systems by the driver's steering model with predictability," Bulletin of the Jan. Soc. of Mech. Eng., vol. 12, no. 51, pp. 495-500, 1969.



Yuichi Saito received the B.S. and M.S. degrees in mechanical engineering from the Shibaura Institute of Technology in 2008, and 2010, and the Doctor's degree in engineering from University of Tsukuba in 2015. Since 2015, he has been with the Tokyo University of Agriculture and Technology, where he is an Assistant Professor at the Department of Mechanical Systems Engineering. His research interests include smart collaborations between human and machine, shared control, and vehicle dynamics.

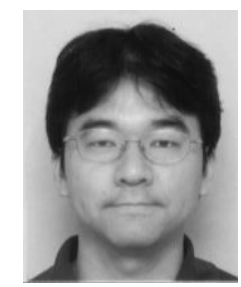

Makoto Itoh received the B.S., M.S., and Doctor's degrees in engineering from University of Tsukuba in 1993, 1995, and 1999, respectively. Since 2013, he has been a Professor with the Faculty of Engineering, Information and Systems, University of Tsukuba. His research interests include enhancement of operator's situation awareness, adaptive automation, and building of appropriate trust as well as prevention of over-trust and distrust.



Toshiyuki Inagaki received the B.S., M.S., and Doctor's degrees in systems engineering from Kyoto University in 1974, 1976, and 1979, respectively. Since 1980, he has been with the University of Tsukuba. Since 2015, he has been Vice President \& Executive Director, University of Tsukuba. His research interests include human-machine symbiosis, evidential reasoning and decision making with incomplete information, reliability and safety of human-machine systems, and human-centered automation. 